\title{
Performance Analysis of MIMO-OFDM System using Space-time Turbo Codes and Adaptive Beamforming
}

\author{
Suchita Varade \\ Assistant Professor \\ Department of Electronics \& \\ Telecommunication Engg. \\ Priyadarshini College of Engineering, \\ Nagpur, India
}

\author{
Kishore Kulat \\ Professor \\ Department of Electronics \& Compute \\ Science. \\ Visvesvaraya National Institute of \\ Technology, Nagpur, India
}

\begin{abstract}
This paper aims at performance analysis of the system which uses space-time turbo codes combined with adaptive beamforming for multiple transmits and receives antennas based orthogonal frequency division multiplexing (OFDM) system. The performance of the proposed technique is tested for two adaptive beamforming algorithms LMS \& LLMS. Simulation results demonstrate that the proposed system not only has good ability of suppressing interference, but also significantly improves the bit-error rate (BER) performance of the system. Experimental results show that an adaptive beamforming gives the optimum performance on AWGN channels. This system will also be optimum on fading channels when combined with space-time turbo codes.
\end{abstract}

\section{Keywords}

LMS (least mean squares), LLMS (LMS-LMS), space-time turbo code (STTC), spatial diversity.

\section{INTRODUCTION}

Recently, the space-time coded orthogonal frequency division multiplexing (STC-OFDM) system is a promising scheme for broadband communications. As wireless communication systems look intently to compose the transition from voice communication to interactive internet data, achieving higher bit rates becomes both increasingly desirable and challenging. Space-time coding (STC) is a communications technique for wireless systems that inhabit multiple transmit antennas and single or multiple receive antennas. Space-time codes make use of advantage of both the spatial diversity provided by multiple antennas and the temporal diversity available with time-varying fading. Space-time codes can be divided into block codes and trellis codes. Space-time trellis coding merges signal processing at the receiver with coding techniques appropriate to multiple transmit antennas. The advantages of space-time codes (STC) make it extremely remarkable for high-rate wireless applications. Initial STC research efforts focused on narrowband flat-fading channels. The decoding complexity of Space-time turbo codes STTC increases exponentially as a function of the diversity level and transmission rate. [1]

Among the existing air-interface techniques, orthogonal frequency division multiplexing (OFDM) [2]-[4] has shown a number of advantages and has attracted substantial interest. New wireless techniques, such as ultra wideband (UWB) [5],advanced source and channel encoding as well as various smart antenna techniques, for example space-time codes (STCs) [6], space division multiple access (SDMA) [1] and beamforming, as well as other multiple-input multiple output (MIMO) [7], [8] wireless architectures are capable of offering substantial gains attaining the maximum possible diversity gain is the objective of the family of space-time block coding (STBC) [11] as well as space- time trellis coding (STTC) [12] schemes found in the literature [6]. The beneficial effects of second-order transmit and up to sixth-order receiver diversity was demonstrated in the context of STBC-aided MIMO-OFDM [13], [14], but space-time coding MIMO will not be considered further in this treatise. Finally, beamforming mitigates the effects of interfering users roaming in the vicinity of the desired user [15], provided that their received signals are angularly separable.

The quality of a wireless link can be described by three basic parameters, namely the transmission rate, the transmission range and the transmission reliability. Conventionally, the transmission rate may be increased by reducing the transmission range and reliability. By contrast, the transmission range may be extended at the cost of a lower transmission rate and reliability, while the transmission reliability may be improved by reducing the transmission rate and range [16]. However, with the advent of MIMO assisted OFDM systems, the above-mentioned three parameters may be simultaneously improved [16]. Initial field tests of broadband wireless MIMO OFDM communication systems have shown that an increased capacity, coverage and reliability is achievable with the aid of MIMO techniques [17] Furthermore, although MIMOs can potentially be combined with any modulation or multiple access technique, recent research suggests that the implementation of MIMO-aided OFDM is more efficient, as a benefit of the straightforward matrix algebra invoked for processing the MIMO OFDM signals [16].

Multi-antenna implementation such as MIMO and beamforming enhances the coverage and capacity in even the most challenging environments. Smart antenna utilizes the strong spatial correlation to process the received signal by antenna arrays with beamforming technique. It is able to provide high directional beamforming gain and reduces the interference from other direction under high spatial correlated MIMO channel.

Considering the advantages of these various MIMO techniques, there is a need to integrate them so that the whole system can benefit from these technologies. These three techniques have the same feature in the view of requiring the multiple antenna elements, but have the contradictive requirement for antenna element spacing. Because it is conflictive that the smart antenna works under high spatial correlated MIMO channel .while the spatial diversity technique work under low spatial correlated MIMO channel.

Thus, the combination of STC and OFDM is a promising scheme for future wideband multimedia wireless communication systems [2]. However, using multiple transmit antennas causes mutual interference either signals transmitted from different antennas of the same transmitter or the other user transmitters [10]- [11].

Antenna array is a spatial filter to suppress the interference while enhancing the desired signals. In [8], it is shown that the scheme of MIMO wireless systems incorporating beamforming methods 
before space-time decoder can effectively mitigate interference while preserving the space-time structure.

Organization of this paper is as follows. Section2 present an Adaptive beamforming and Space-Time Block Coding in MIMO Using OFDM .Section 3 presents adaptive beamforming algorithms LMS \& LLMS Whereas simulation results are given in Section 4 followed by the conclusions. In this paper the symbols [.] ${ }^{*},[.]^{\mathrm{T}}$, and, [.] ${ }^{\mathrm{H}}$, are conjugate, transpose and, conjugate transpose operators, respectively.

\section{ADAPTIVE BEAMFORMING AND SPACE-TIME TURBO CODING IN MIMO USING OFDM}

\subsection{System model}

Fig.1 presents the block diagram of the MIMO OFDM spacetime coded system with smart antenna. The OFDM transmission and reception are respectively presented in Fig.1 (a) \& Fig.1 (b).

\subsection{Transmitter Part}

At transmitter first, the data is generated from a random source, consists of a series of ones and zeros. Data input bits are converted into symbol vector using modulation. Modulation scheme used to map the bits to symbols are BPSK, QPSK, 16 PSK, 256 PSK. The data modulated symbol sequence is fed into the ST Turbo coder. The ST Turbo coder maps the block of mbinary symbols into two sequences. The first sequence is generated using normal convolution code and second is generated using interleaved convolution code. The two output signals from STTC $\mathrm{x} 1$ and $\mathrm{x} 2$ termed as normal sequence \& interleaved signal as shown in fig.(1).

Since the transmission is done block wise, when forward error correction (FEC) is used, the size of the data generated depends on the block size used, modulation scheme used to map the bits to symbols (BPSK,QPSK16 PSK,256 PSK), and whether FEC is used or not [1]. The generated data is passed on to the next stage i.e to the FEC block

Forward error correcting codes are applied to normal convolution code sequence and interleaved convolution code sequence. The error correcting codes are used, to avoid long run of zeros or ones, as the data generated is randomized. This results in ease in carrier recovery at the receiver. The randomized data is encoded using tail biting convolution codes (CC) with a coding rate of $1 / 2$

\subsection{Subcarrier allocation:}

The subcarrier allocation separates data into set of 4 subcarriers and OFDM time symbol then passed onto the next stage, the IFFT, to convert into time domain.

\subsection{IFFT :}

An efficient way of implementing IDFT is by inverse fast Fourier transform (IFFT).

$$
\operatorname{Sj}(n)=\frac{1}{N} \sum_{n=0}^{N-1} x_{j} \exp \left(\frac{j 2 \pi k n}{N}\right)
$$

Hence IFFT is used in generation of OFDM symbol. The IFFT size ('N' value) is considered as 256 in simulations. This data is fed to the channel which represents AWGN model. The resulting signal at the transmitter can be expressed as

$$
x(n)=\frac{1}{N} \sum_{n=0}^{N-1} X(k) \exp \left(\frac{j 2 \pi k n}{N}\right)
$$

Where, $\mathrm{j}$ is the number of transmitting antennas.

\subsection{Channel}

In NLOS wireless communication, the received signal is a combination of many multipath signals, which are result of reflections from surrounding objects. These multipath have different amplitude and phase and may add either constructively or destructively leading to a complex envelope, i.e. fading. Fading characteristics depend on the channel parameters (rms delay spread and Doppler spread) and signal parameters (symbol period and bandwidth). Multipath delay spread leads to time dispersion and frequency selective fading and Doppler spread leads to frequency dispersion and time selective fading.

The two transmitted signals are scattered using 20 scattered sequences. Twenty random sequences are generated with the noise level as 10 . Finally additive white Gaussian noise (AWGN) is added as a last component in the channel.

\subsection{Receiver}

The MIMO OFDM receiver is shown in Fig. 1(b). At the receiver, the signals are received and combined with different path loss and different fading fluctuation. Receiving antennas used at the receiver are 2 . The complex-valued propagation channel gains experienced between the two transmit antennas \& two receiving antennas is represented by $\mathrm{H}$. The received Signal from $\mathrm{j}$ transmitting antenna and $\mathrm{P}$ propagation paths is expressed as,

$$
V(n)=\sum_{p=1}^{P} \sum_{j=1}^{2}\left[A \otimes H^{p}\right] S_{j}(n-p \Delta \tau)+G(n)
$$

The first thing done at receiver (in simulation) is estimation the angle of reception.

1. Find out the sum of power of all incident signals for each angle from $\left(-100^{\circ}\right.$ to $\left.100^{\circ}\right)$ of all elements. (As the sequences are scattered.)

2. From the maximum peak in the power spectrum we can estimate the DOA's of the desired signals.

3. Two transmitting \& two receiving antennas are used. So at the receiver we get four copies of the received signal.

4. Two copies form normal sequences and two from interleaved sequence.

5.At the receive these two copies termed as normal angle reception signals i.e when the signal from transmitting antenna 1 $\mathrm{Tx} 1$, is received by the receiving antenna $1, \mathrm{Rx} 1$.Same is the case for Rx2.So, two signals are obtained normal angle reception signal $1 \&$ normal angle reception signal 2 .Now when reciving antenna $1 \mathrm{Rx} 1$, received the signal from Transmitiing antenna 2 Tx2 it is termed as mixed angle reception. Same is the case for $\mathrm{Rx} 2$. So, two signals are obtained mixed angle reception signal 1 $\&$ mixed angle reception signal 2 . 


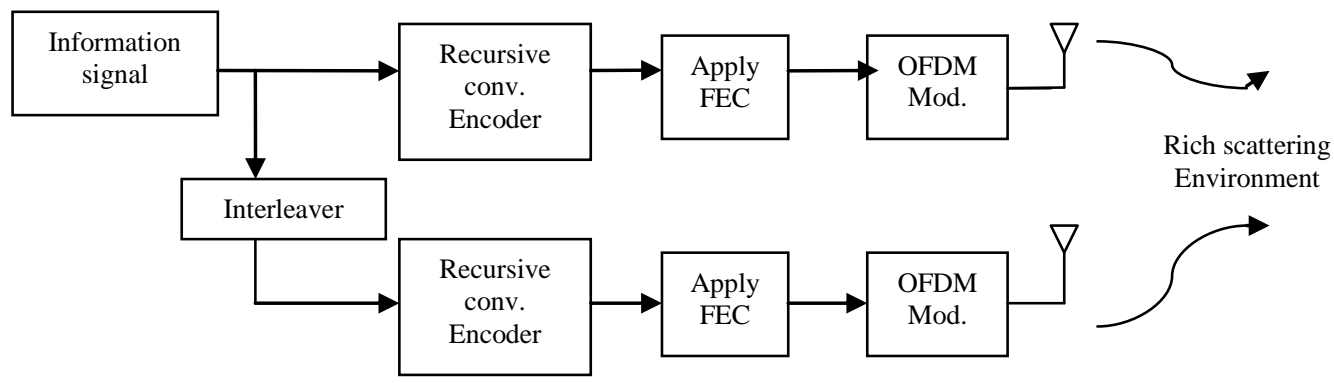

Fig.1 (a) MIMO OFDM Transmitter

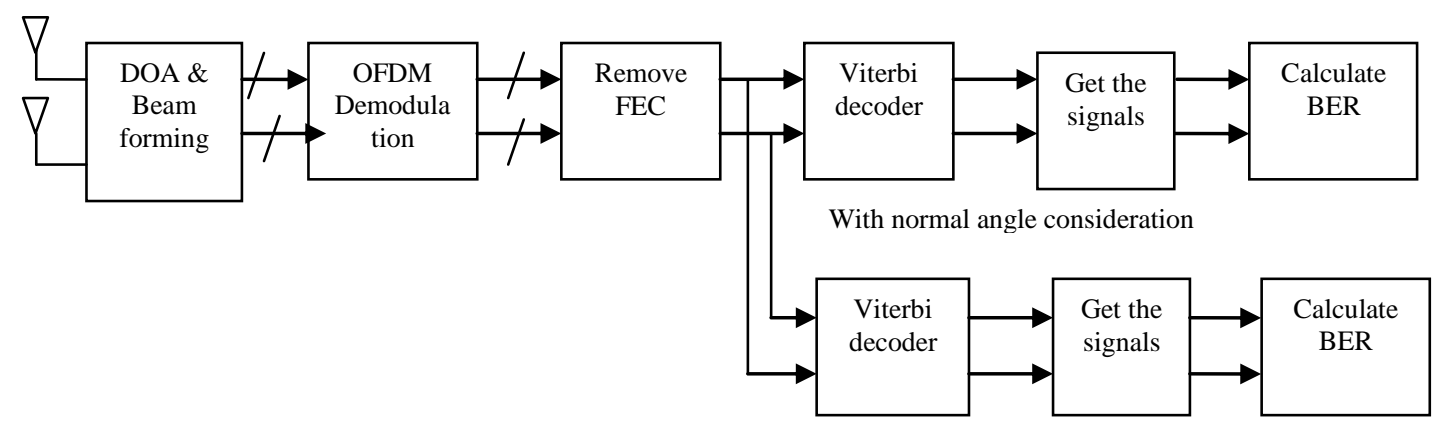

With mixed angle consideration

Fig.1 (b) MIMO OFDM Receiver

Fig.1: Block diagram of ST-TURBO CODED MIMO- OFDM system using Adaptive beamforming at receiver

6. After estimating the angle of arrival an Adaptive beamforming algorithms LMS \& LLMS are used to minimize the interference. These algorithms are described in section III.

\subsection{FFT}

After receiving four copies of the signal, signals are fed to the FFT for frequency domain transformation.

\subsection{Removal of FEC}

After this FEC is removed from the normal angle reception signals \& mixed angle reception signals.

\subsection{Viterbi Decoder}

For decoding of the signals viterbi decoders are used. Four sequences are compared to find out which sequence has minimum value of error in bits. For this each sequence is matched with the input sequence to find out which sequence has minimum BER. Finally it gives the result in terms of recovered sequence $\&$ recovered mode i.e. normal reception mode or mixed reception mode along with the angle of recovery.

\section{ADAPTIVE BEAMFORMING}

This section describes adaptive beamforming algorithms LMS \& LLMS algorithm. Because of its simplicity and robustness, the LMS algorithm has become one of the most popular adaptive signal processing techniques adopted in many applications including antenna array beamforming. Moreover, there is always a tradeoff between the speed of convergence of the LMS algorithm and its residual error floor when a given adaptation step size is used. LLMS is the new adaptive algorithm, which employs two Least Mean Square (LMS) sections in tandem.[20]

\subsection{LMS-based adaptive beamforming}

\section{Algorithm}

Firstly, we consider the LMS-based adaptive beamforming methods. The LMS algorithm is a popular solution used in beamforming technique. This algorithm is easy to implement with low computation and performs pretty well. The basic LMS algorithm is expressed as follow [8]

$$
w(n+1)=w(n)+2 \mu x(n) e(n)
$$

The reference signal d (t) generated at the receiver is usually assumed to have similar statistical properties as the transmitted signal.

For the purpose of simulation, we will simply assume that the reference signal is identical to the incoming signal. The error signal e (n) $=d(n)-y(n)$ is fed into the weight updating algorithm. The criterion for determining the weights is based on minimizing the mean squared error (MSE) between the beamformer output and the reference signal:

$$
\begin{aligned}
& E\left(e^{2}\right)=E\left[(d-y)^{2}\right] \\
& \left.E\left(e^{2}\right)=E\left(d-w^{H} x\right)^{2}\right] \\
& E\left(e^{2}\right)=E\left(d^{2}\right)-2 E\left(d w^{H} x\right)-E\left[\left(w^{H} x\right)^{2}\right] \\
& E\left(e^{2}\right)=E\left(d^{2}\right)-2 w^{H} r-w^{H} R_{x x} w
\end{aligned}
$$

Where $\operatorname{Rxx}$ is the autocorrelation matrix of the received signal $\mathrm{x}$ and $\mathrm{r}=\mathrm{E}[\mathrm{dx}]$ is the cross-correlation between the reference signal and the received signal. The MSE surface is a quadratic function 
of $\mathrm{w}$ and is minimized by setting its gradient with respect to $\mathrm{w}$ to zero.

$$
\Delta w\left[E\left(e^{2}\right)\right]=-2 r+2 R_{x x} w=0
$$

Yielding the well-known Wiener-Hopf solution-

$$
w_{o p t}=R_{x x}^{-1} r
$$

The LMS algorithm is a stochastic gradient optimization algorithm that converges to this solution. It is based on a traditional optimization technique called the Method of Steepest Descent. The weight vector is made to evolve in the direction of the negative gradient which points towards the minimum.

$$
\begin{gathered}
w(n+1)=w(n)+\frac{\mu}{2}\left\{-\nabla w\left[E\left(e^{2}(n)\right)\right]\right\} \\
w(n+1)=w(n)+\frac{\mu}{2}\left\{2 r(n)-2 R_{r r}(n) w(n)\right\}
\end{gathered}
$$

Where $\mathrm{R}_{\mathrm{xx}}$ and $\mathrm{r}$ are given by

$$
\begin{gathered}
R_{x x}(n)=x(n) x^{H}(n) \\
r(n)=d(n) x(n)
\end{gathered}
$$

This gives us a simple expression for weight updating:

$$
w(n+1)=w(n)+2 \mu x(n) e(n)
$$

The LMS algorithm is initiated with an arbitrary value $\mathrm{w}(0)$ for the weight vector at $n=0$. The successive of the weight vector eventually leads to the minimum value of the mean squared error. LMS algorithm can be summarized in following equations:

$$
\begin{gathered}
\text { Output, } y(n)=w^{H}(n) x(n) \\
\text { Error, } e(n)=d(n)-y(n) \\
\text { Weight, } w(n+1)=w(n)+\mu x(n) e^{*}(n)
\end{gathered}
$$

\subsection{LLMS-based adaptive beamforming}

The LLMS algorithm is shown in Fig. 2[20].The intermediate output, LMS1 yielded from the first LMS section, LMS1, is multiplied by the image array factor $\left(\mathrm{A}^{\prime}\right)$ of the desired signal. The resultant "filtered" signal is further processed by the second LMS section, LMS2. For the adaptation process, the error signal of LMS2, $e_{2}$, is fed back to combine with that of LMS1, to form the overall error signal, $e_{\text {LLMS }}$, for updating the tap weights of LMS1. As shown in Fig.2, a common external reference signal is used for both the two LMS sections, i.e., $\mathrm{d} 1$ and $\mathrm{d} 2$.The error signal for updating LLMS1 at the $\mathrm{j}^{\text {th }}$ iteration is given by

$$
\begin{aligned}
& e_{L L M}(j)=e_{1}(j)-e_{2}(j-1) \\
& e_{1}(j)=d_{1}(j)-w_{1}^{H}(j) X_{1}(j) \\
& e_{2}(j)=d_{2}(j)-w_{2}^{H}(j) X_{2}(j)
\end{aligned}
$$

The input signal of LMS2 is derived from the LMS1, such that

$$
X_{2}(j)=A^{\prime} y_{L M S 1}(j)=w_{1}^{H}(j) X_{1}(j)
$$

Where $\boldsymbol{A}$ 'is the image of the array factor of the desired signal. The weight vector $\mathrm{W}$ for the $\mathrm{i}^{\text {th }}$ LMS section is updated according to

$$
w_{i}(j+1)=w_{i}(j)+\mu_{i} e_{i}(j) X_{i}(j)
$$

Where, $0<\mu_{i}<\mu_{0} \& i$ is 1 for LMS1 and 2 for LMS2. $\mu_{\mathrm{i}}$ is the step size and $\mu_{0}$ is a positive number that depends on the input signal statistic.

\section{SIMULATION RESULTS}

In this section, the computer simulations investigate the BER performance of the proposed technique for Space Time Turbo Coded-OFDM system. Various simulation parameters assumed are,
1. Code length is 1000
2. Number of carriers $=64$
3. Window type Hamming
4. Guard type-No guard type, zero level guard period, cyclic extension of end of symbols, cyclic extension of end of symbols with first half of guard period is zero.

The system model has been tested for BPSK, QPSK, 16 PSK, 256 PSK modulations with an AWGN channel. The simulations as shown in Fig. 4 are based on both LMS and LLMS algorithm. In the simulation, there are 2 transmitter antennas and the number of the receiving antennas is 2 . The DOAs of two transmitter antennas for desired user are $10^{\circ}, 40^{\circ}$. It has been observed that when the signals are scattered then it changes the angle of arrival i.e. the DOA's in this case are not $10^{\circ} \& 40^{\circ}$ but at some other angles. The simulation result of Fig. $4 \mathrm{v}$ ) shows the BER comparison of proposed technique based on LMS \& LLMS algorithm. 


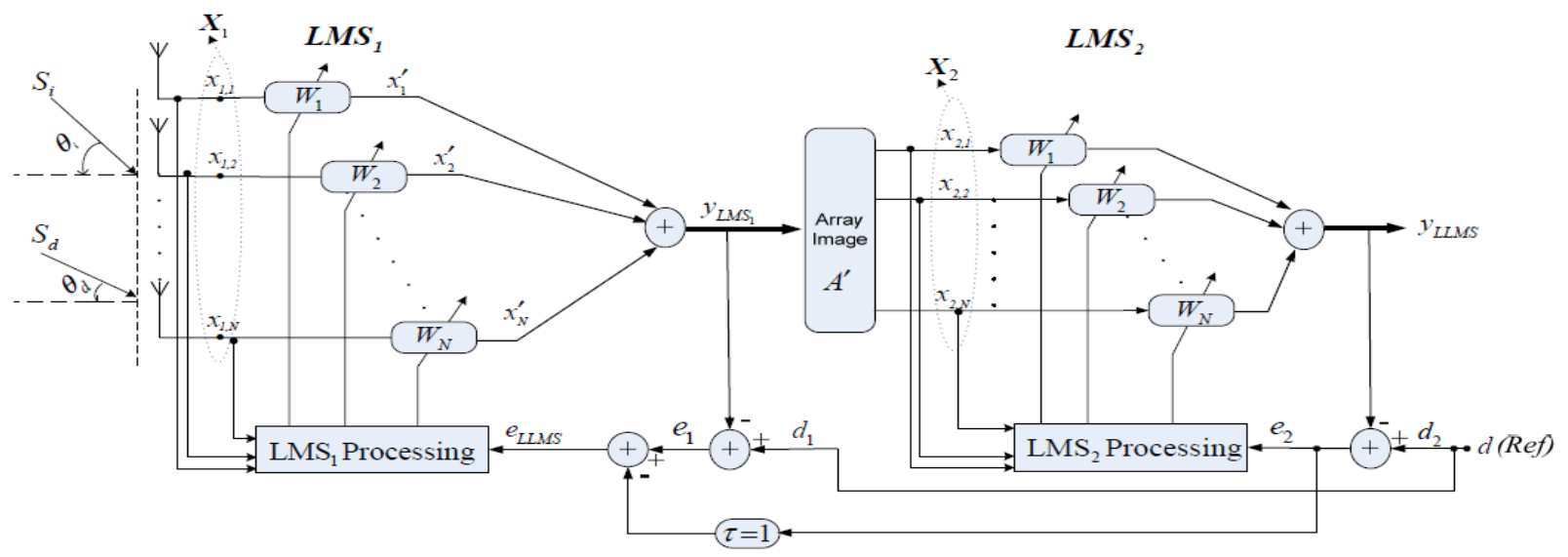

Fig.2 LLMS algorithm [as proposed in Reference 20]

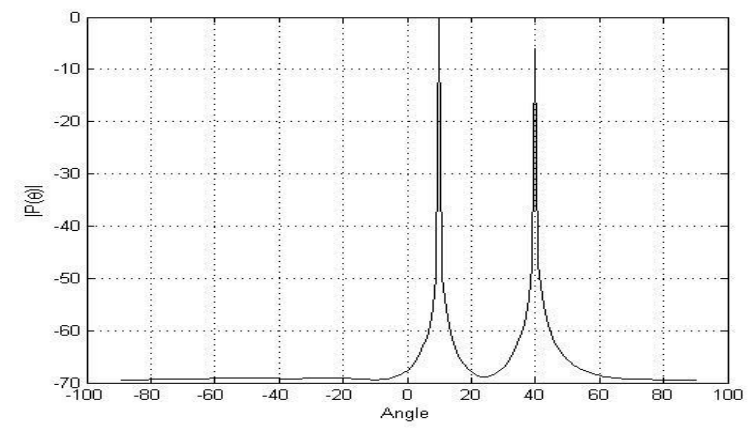

Fig.4 i) Power spectrum for angle -100 to100 (Max. peak at

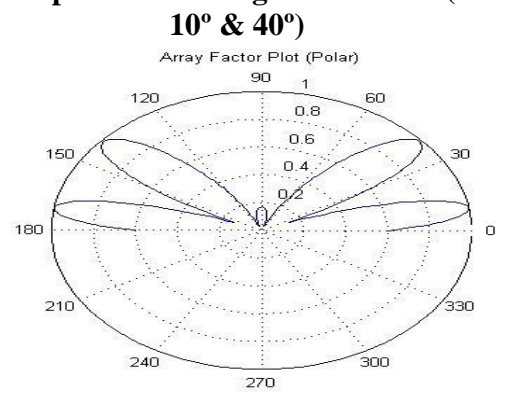

Fig.4 ii) Polar plot (Beam at $\left.10^{\circ} \& 40^{\circ}\right)$

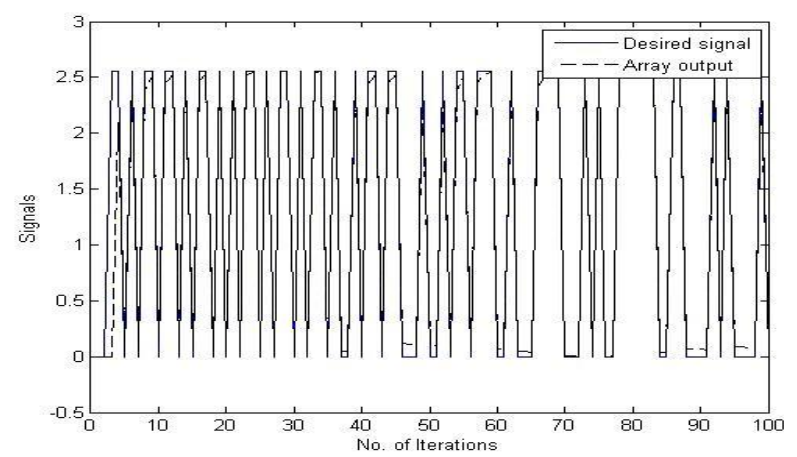

Fig.4 iii) Desired signal \& array output
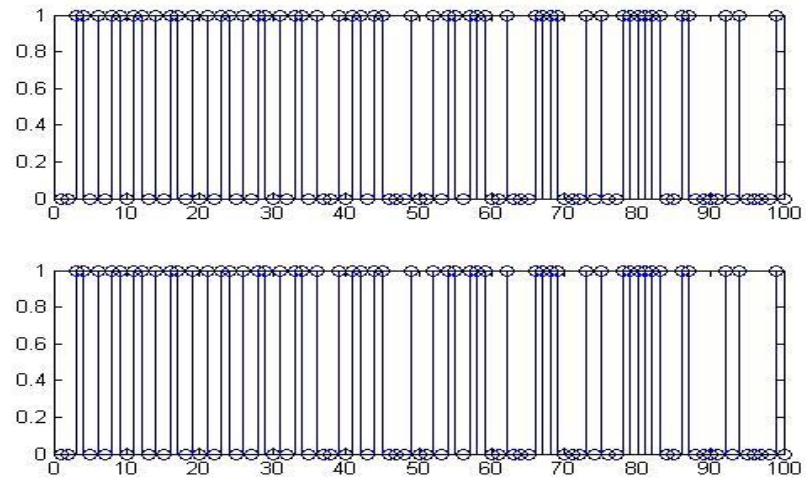

Fig.4 iv) Input sequence \& Output sequence
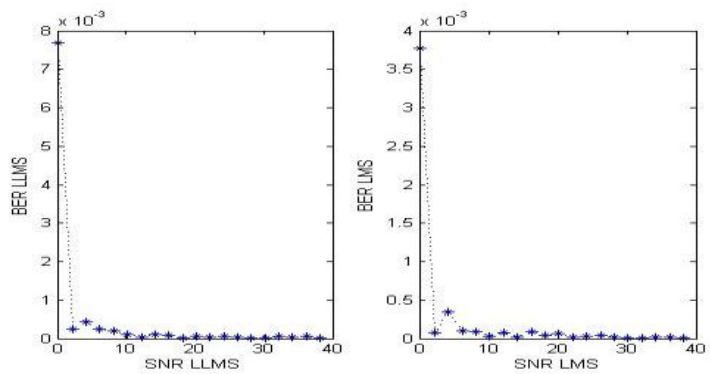

Fig.4 v) BER plot for LLMS \& LMS for BPSK modulation
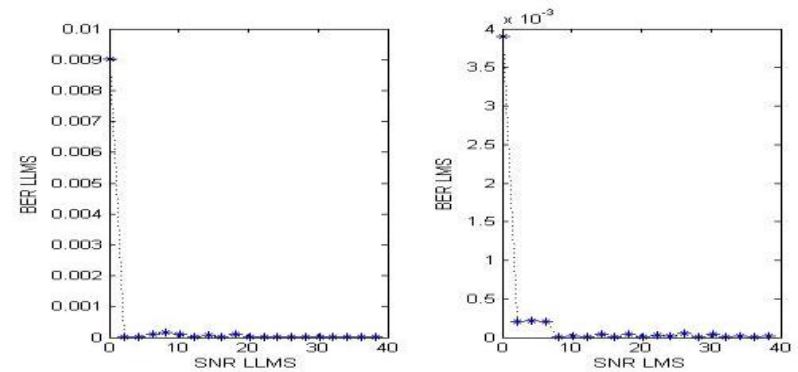

Fig.4 v) BER plot for LLMS \& LMS for QPSK modulation 

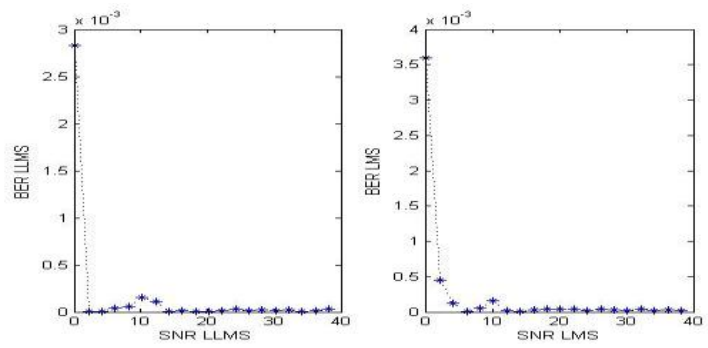

Fig.4 v) BER plot for LLMS \& LMS for 16-PSK modulation
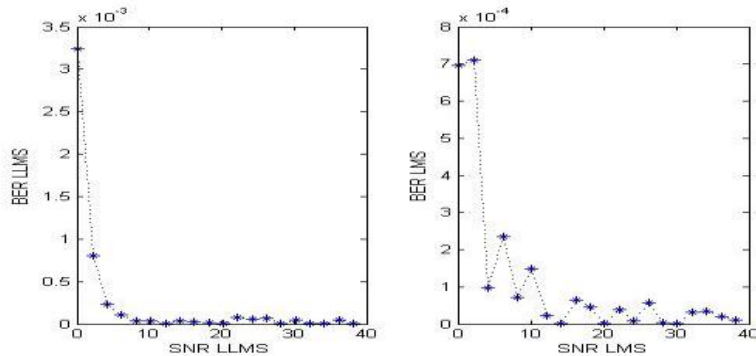

Fig.4 v) BER plot for LLMS \& LMS for 256-PSK modulation

\section{CONCLUSION \& FUTURE WORK}

The proposed system has been verified in AWGN channel with scattering. The results show that a proposed system can more effectively mitigate interference. The use of LMS \& LLMS adaptive beamformer in the STC-OFDM system also enhances the system performance in terms of BER. The BER performance of the system with LLMS algorithm is better than the system with LMS algorithm. It can be concluded that the proposed method not only has the good ability of suppressing interference, but significantly improves the bit-error rate (BER) performance for STC-OFDM system.

Future work should focus on testing of the system for different channel conditions. Furthermore, OFDMA can be used to get a MIMO-MU (multiuser) system.

\section{REFERENCES}

[1] C. V. Seshaiah, S. Nagarani "A Survey on Space-Time Turbo Codes" IJCSIS, Vol. 7 No. 3, March 2010, 171177, ISSN 1947 5500J. Clerk Maxwell, A Treatise on Electricity and Magnetism, 3rd ed., vol. 2. Oxford: Clarendon, 1892, pp.68-73.

[2] L. Hanzo, S. X. Ng, T. Keller, and W. T. Webb, Quadrature AmplitudeModulation: From Basics to Adaptive TrellisCoded, Turbo-Equalised and Space-Time Coded OFDM, CDMA and MC-CDMA Systems,3rd ed. Piscataway, NJ: IEEE Press/Wiley,2004.

[3] R. V. Nee and R. Prasad, OFDM for Wireless Multimedia Communications. London, U.K.:Artech House, 2000.

[4] J. A. C. Bingham, BMulticarrier modulation for data transmission: An idea whose time has come,[ IEEE Commun. Mag., vol. 28,no. 5, pp. 5-14, May 1990.

[5] W. C. Chung, N. J. Aug., and D. S. Ha, BSignaling and multiple access techniques for ultra wideband $4 \mathrm{G}$ wireless communication systems Wireless Commun., vol. 12, pp. 46-55, Apr. 2005

[6] L. Hanzo, T. H. Liew, and B. L. Yeap,Turbo Coding, Turbo Equalisation and Space-Time Coding for Transmission Over Fading Channels. Piscataway, NJ: IEEE Press/Wiley, 2002.
[7] J. H. Winters, B Optimum combining in digital mobile radio with with cochannel interference,[ IEEE J. Sel. Areas Commun.,vol. SAC-2, no. 4, pp. 528-539, Jul. 1984

[8] J. H. Winters, BOn the capacity of radio communication systems with diversity in a Rayleigh fading environment, IEEE J. Sel. Areas Commun., vol. SAC-5, no. 5,pp. 871878, Jun. 1987

[9] S. Ng and L. Hanzo, BOn the MIMO channelcapacity of multi-dimensional signal sets, [ in Proc. 60th IEEE Conf. Vehicular Technology,Sep. 2006, vol. 3, pp. 1594-1598.

[10] L. Hanzo, M. Mu"nster, B. J. Choi, and T. Keller, OFDM and MC-CDMA for Broadband Multi-User Communications, WLANs and Broadcasting. Piscataway, NJ:IEEE Press/Wiley, 2003 [11] S. M. Alamouti, BA simple transmit diversity technique for wireless communications,[IEEE J. Sel. Areas Commun., vol. 16, no. 8,pp. 1451-1458, Oct. 1998.

[11] V.Tarokh, A. Naguib, N. Seshadri, and A. R. Calderbank, BSpace time codes for high data rate wireless communication: Performance criteria in the presence of channel estimation errors, mobility, and T. multiple paths, IEEE Trans.Commun.,vol. 47, no. 2, pp. 199-207, Feb1999

[12] T.-H. Liew and L. Hanzo, BSpace-time trellis and spacetime block coding versus adaptive modulation and coding aided OFDM for wideband channels, IEEE Trans. Veh.Technol., vol. 55, no. 1, pp. 173-187, Jan. 2006

[13] J. Blogh and L. Hanzo. (2002).Third-Generation Systems and Intelligent Networking. Piscataway, NJ, IEEE Press/Wiley. [Online]. Available: http://wwwmobile.ecs.soton.ac.uk

[14] Datacomm Research Company, Using MIMO-OFDM Technology to Boost Wireless LAN Performance Today, White Paper,St. Louis, MO, Jun. 2005.

[15] H. Sampath, S. Talwar, J. Tellado, V. Erceg,and A. J. Paulraj, BA fourth-generation MIMO-OFDM broadband wireless system:Design, performance, and field trial results, IEEE Commun. Mag., vol.40, no.9,pp. 143-149, Sep. 2002.

[16] V. Tarokh, N. Seshadri, and A. R. Calderbank, Sspace-time block codes from orthogonal designs", IEEE Trans. on IT, Vol. 45, pp. 1456-1467, July 1999

[17] S. M. Alamouti, "A simple transmit diversity technique for wireless Communications", IEEE JSAC, Vol. 16, No. 8, pp. 1451-1458, October 1998.

[18] G.Jongren,M.Skoglund and B. Ottersten, "Combining beamforming and orthogonal space-time block coding," IEEE Trans. Inf. Theory, vol. 48, pp. 611-627, Mar. 2002.

[19] V. Tarokh, H. Jafarkhani, A.R. Calderbank, "Space-time block coding for wireless communications: Performance results", IEEE Journal on Selected Areas in Communication,vol.17, pp. 451-460, Mar.

[20] Jalal Abdul sayed SRAR, Kah-Seng CHUNG and Ali MANSOUR "Adaptive Array Beamforming using a Combined LMS-LMS Algorithm" IEEEAC paper\#1606, Version 2, Updated 2009:12:27

[21] S.W.Varade \& K.D.Kulat "Robust Algorithms for DOA Estimation and Adaptive Beamforming for Smart Antenna Application" International Conference on Emerging Trend in Engineering \& Technology (ICETET 2009) at G.H.Raisoni College of Engg.,Nagpur,16-18 Dec.2009

[22] S.W.Varade \& K.D.Kulat "Performance Analysis of MVDR Beamformer for Smart Antenna Applications" International Conference on VLSI and Communication(ICVCom-09), Kerela April 16-18, 2009 